\title{
DESSORÇÃO IÔNICA E DEGRADAÇÃO DE FILMES DE POLIPIRROL DOPADO COM DODECILSULFATO INDUZIDAS POR ELÉTRONS DE ALTA ENERGIA
}

Caroline Arantes e Maria Luiza M. Rocco*

Departamento de Físico-Química, Instituto de Química, Universidade Federal do Rio de Janeiro, Cidade Universitária, Ilha do Fundão, 21941-590 Rio de Janeiro - RJ, Brasil

Antonio Gerson Bernardo da Cruz e Ana Maria Rocco

Escola de Química, Universidade Federal do Rio de Janeiro, Cidade Universitária, Ilha do Fundão, 21949-900 Rio de Janeiro - RJ, Brasil

Recebido em 30/11/06; aceito em 21/6/07; publicado na web em 19/12/07

\begin{abstract}
IONIC DESORPTION AND DEGRADATION OF POLYPYRROLE FILMS DOPED WITH DODECYLSULFATE INDUCED BY HIGH ENERGY ELECTRONS. Electron stimulated ion desorption (ESID) and degradation studies of polypyrrole doped with dodecylsulfate (PPy/DS) deposited on FTO were performed using time-of-flight mass spectrometry (TOF-MS) for ion analysis. The results suggest a strong contribution from fragments of the dodecylsulfate hydrocarbon chain to the mass spectra. In the 650$1500 \mathrm{eV}$ energy range the ion yield curves show maxima at about 600,1200 and $1400 \mathrm{eV}$, which can be related to carbon, nitrogen and oxygen-containing fragments, respectively, and interpreted in terms of the Auger Stimulated Ion Desorption (ASID) mechanism. Degradation studies indicate rapid loss of heavier hydrocarbons and an increase of bulk and substrate fragments. Some degradation profiles suggest formation of new species.
\end{abstract}

Keywords: electron stimulated ion desorption; polypyrrole films; degradation.

\section{INTRODUÇÃO}

O polipirrol (PPy) é um dos polímeros intrinsecamente condutores (PIC) que mais tem atraído a atenção de pesquisadores, tanto do ponto de vista fundamental quanto aplicado, devido a sua alta condutividade, estabilidade química e síntese relativamente simples. Suas propriedades redox, ópticas e elétricas os tornam potenciais para diversas aplicações, tais como, dispositivos eletrocrômicos $^{1}$, baterias ${ }^{2}$, capacitores ${ }^{3}$ e sensores ${ }^{4}$, abrindo novas perspectivas para as áreas de nanociência e nanotecnologia.

Filmes de PPy podem ser obtidos na forma oxidada, através de síntese eletroquímica, com a incorporação de contra-íons para manter a eletro-neutralidade do sistema. As propriedades do material são fortemente influenciadas pela natureza do contra-íon incorporado, tornando possível sua modulação de acordo com a aplicação desejada. Uma grande vantagem da síntese eletroquímica é a obtenção do material na forma de filmes auto-suportados sobre um substrato condutor, o que possibilita a realização de diversas aplicações e também estudos de superfícies.

A síntese e caracterização de filmes de PPy dopados com diferentes contra-íons têm sido realizadas através de diferentes técni$\operatorname{cas}^{5-8}$. Entretanto, estudos da interação destes materiais com elétrons ou fótons ainda são muito pouco explorados ${ }^{9-11}$. Os PICs podem ser expostos a estes tipos de radiação quando utilizados na fabricação de dispositivos eletrônicos ou durante processos litográficos $^{12,13}$. Assim, é de grande importância a compreensão dos processos que originam a fragmentação e a degradação destes materiais quando expostos a um feixe de elétrons ou fótons, uma vez que estes podem causar modificações significativas nas propriedades físicas e químicas destes materiais ${ }^{10,14}$. Estudos da interação de elétrons e fótons de alta energia com filmes poliméricos têm se tornado alvo de grande interesse, surgindo como uma im-

*e-mail: luiza@iq.ufrj.br portante área de pesquisa ${ }^{15-19}$. Entretanto, poucos grupos têm concentrado esforços para a compreensão detalhada dos processos originários destas interações. Até o presente momento, os estudos da interação de elétrons e fótons com polímeros condutores baseiamse nas alterações das propriedades físicas e químicas do material devido à irradiação, que são verificadas através de técnicas eletroquímicas e morfológicas ${ }^{10,20,21}$.

A técnica de dessorção iônica induzida por feixe de elétrons ou fótons pode fornecer informações importantes com relação aos processos de fragmentação de diferentes polímeros ${ }^{17,18,22}$. Acredita-se que o principal processo que governa a dessorção iônica nestes materiais quando expostos à radiação de alta energia é o processo Auger, que pode ser descrito de forma simplificada como um processo de relaxação decorrente da excitação (Auger Ressonante) ou ionização (Auger Normal) de um elétron de camada interna. Após a emissão do elétron Auger, ocorre a formação de buracos em orbitais de valência que levam à fragmentação e dessorção de íons positivos, devido à repulsão coulômbica entre os buracos formados (explosão coulômbica), dando origem ao mecanismo conhecido como ASID ("Auger Stimulated Ion Desorption").

Derivados do politiofeno foram estudados utilizando-se técnicas de dessorção iônica através da interação com fótons ${ }^{22}$ e elétrons ${ }^{17}$. O poli(3-metiltiofeno) foi estudado através da técnica de dessorção iônica estimulada por fótons (PSID - "Photon Stimulated Ion Desorption"), apresentando fotofragmentação seletiva quando irradiado com fótons de energia correspondente à borda $\mathrm{K}$ do enxofre $(\sim 2400 \mathrm{eV})^{22}$. O efeito da irradiação do filme de poli(3hexiltiofeno) com elétrons de baixa energia foi estudado através de fluorescência, XPS, EELS e ESID de íons positivos e negativos, utilizando-se elétrons primários de $0,5 \mathrm{keV}^{17}$.

Gazotti e colaboradores ${ }^{11}$ realizaram estudos de degradação de filmes de PPy/DS através de aquecimento a $100^{\circ} \mathrm{C}$ e por irradiação simulando a luz solar. A ação térmica causou perda de condutividade e diminuição gradual das correntes de pico anódico e catódico que 
foram medidas por voltametria cíclica. Já a ação da radiação solar não alterou de maneira significativa as propriedades do material.

Neste trabalho, foram realizados estudos de dessorção iônica e degradação de filmes de PPy/DS através da técnica de ESID ("Electron Stimulated Ion Desorption"), utilizando-se elétrons de alta energia e espectrometria de massa por tempo-de-vôo para análise dos íons formados.

\section{PARTE EXPERIMENTAL}

Os filmes de PPy/DS foram obtidos através de deposição galvanostática em um eletrodo de vidro recoberto com FTO (óxido de estanho dopado com flúor) da Flexitec, com dimensões de $25 \mathrm{x}$ 10 x $1 \mathrm{~mm}$ e resistência de 10 a $15 \Omega / \square$. A síntese foi realizada em uma célula de um compartimento, utilizando-se eletrodo de platina com dimensões de $25 \times 10 \times 0,5 \mathrm{~mm}$ como contra-eletrodo e eletrodo de calomelano saturado (ECS) (Analion) como eletrodo de referência. A densidade de corrente utilizada foi de $0,1 \mathrm{~mA} \mathrm{~cm}^{-2}$ e os tempos de crescimento dos filmes de diferentes espessuras foi de $6 \mathrm{e}$ 40 minutos $(\mathrm{Q}=36$ e $240 \mathrm{mC}$, respectivamente) a uma temperatura de $5^{\circ} \mathrm{C}$, utilizando-se solução aquosa de $0,05 \mathrm{~mol} \mathrm{~L}^{-1}$ de pirrol destilado (Aldrich) e 0,05 $\mathrm{mol} \mathrm{L}^{-1}$ de dodecilsulfato de sódio $98 \%$ (NaDS - Aldrich) degasada e mantida sob atmosfera de argônio. Foi utilizado um potenciostato/galvanostato Autolab PGSTAT 30 controlado pelo software General Purpose Electro-chemical System (GPES) em todos os experimentos eletroquímicos. As espessuras dos filmes de PPy/DS, calculadas através da Equação de Wernet e Wegner ${ }^{23}$, apresentaram valores iguais a 0,29 e $1,90 \mu \mathrm{m}$ para os filmes crescidos nos tempos de 6 e $40 \mathrm{~min}$, respectivamente. Como controle, o sal do contra-1́on, dodecilsulfato de sódio (NaDS), foi depositado mecanicamente em um substrato de cobre recoberto com prata.

Os estudos de dessorção iônica induzida por elétrons foram realizados em uma câmara de ultra-alto-vácuo (UHV) mantida sob uma pressão da ordem de $10^{-9}$ Torr, que contém um manipulador de amostras X-Y-Z comercial, um canhão de elétrons de energia variável (Kimball Physics - ELG-2) e um espectrômetro de massa do tipo tempo-de vôo (TOF-MS), projetado e construído em nosso laboratório ${ }^{24}$. Este dispositivo consiste de um sistema eletrostático de extração de íons, uma lente colimadora, um tubo de vôo de 25 $\mathrm{cm}$ e um detector de íons composto por um par de placas de microcanais (MCP), dispostos na configuração chevron ${ }^{25}$. O sinal de saída do detector é processado mediante o uso de um sistema padrão de contagem de pulsos, constituído de um pré-amplificador e um discriminador, sendo este sinal usado como sinal de parada (stop) da experiência. O sinal de inicialização (start) é fornecido pelo gerador de pulsos que controla o canhão de elétrons. Estes sinais são enviados a um conversor tempo-digital (TDC - "time to digital converter") para serem acumulados. Para cada pulso de elétrons enviado pelo canhão de elétrons para o alvo, um sinal lógico (start) é enviado do gerador de pulsos para a placa TDC. Os sinais correspondentes do detector (stop) também são enviados para o conversor, que mede o intervalo de tempo entre o sinal de start e os correspondentes sinais de stop, sendo processados por um computador na forma de um espectro de tempo-de-vôo, que apresenta o número de contagens de cada íon detectado em função do seu tempo de vôo, desde a sua formação até detecção. A resolução temporal da placa TDC empregada é de 1 ns/canal. O filme de PPy/DS e o NaDS foram irradiados com um feixe de elétrons pulsado com largura de pulso de $20 \mathrm{~ns}$ e com uma freqüência de $80 \mathrm{kHz}$. A faixa de energia incidente utilizada variou de $650-2900 \mathrm{eV}$ e corresponde à energia nominal do canhão de elétrons mais a diferença do potencial aplicado entre o catodo do canhão de elétrons e o suporte da amostra. $\mathrm{O}$ feixe de elétrons incide sobre a amostra a $60^{\circ} \mathrm{com}$ relação à normal a superfície, estando a amostra posicionada a 10 $\mathrm{mm}$ da entrada do espectrômetro de massa do tipo tempo-de-vôo.

Os valores de potenciais aplicados nos diversos elementos do espectrômetro (grade, lente, tubo-de-vôo e detector) foram simulados através do programa SIMION 3D 6.026. Todas as simulações foram realizadas considerando-se que as espécies dessorvem da amostra em ângulos de $0 \mathrm{a} \pm 90^{\circ}$ e com energia cinética de $0 \mathrm{a} 10 \mathrm{eV}$.

No estudo de degradação, o filme foi irradiado com feixe contínuo de elétrons com energia de $2,2 \mathrm{keV}$ e analisado através do método acima descrito, utilizando-se feixe de elétrons pulsado com energia de $2,2 \mathrm{keV}$.

A atribuição das massas no espectro de tempo-de-vôo foi feita através da simulação dos tempos de vôo dos íons com o programa SIMION 3D 6.0 e utilização da Equação 1 e dois picos conhecidos no espectro

$\mathrm{TOF}=\mathrm{A}(\mathrm{m} / \mathrm{q})^{1 / 2}+\mathrm{B}$

onde TOF é o tempo de vôo de um dado íon, m/q é a relação massa/ carga para este íon e A e B são constantes empíricas calculadas através da medida do tempo de vôo de duas espécies de massas conhecidas. As curvas de rendimento iônico relativo foram obtidas em função da energia dos elétrons incidentes e em função do tempo de exposição ao feixe contínuo de elétrons para os estudos de degradação.

\section{RESULTADOS E DISCUSSÃO}

Na Figura 1, são apresentados espectros de tempo-de-vôo típicos de íons positivos dessorvidos do filme de PPy/DS com espessura de 1,9 $\mu \mathrm{m}$ depositado sobre FTO (Figura 2a) e de dodecilsulfato de sódio (NaDS) (Figura 2b). Os espectros foram obtidos utilizando-se um feixe de elétrons de $2,2 \mathrm{keV}$ de energia e cobrindo uma faixa de massa de 1-100 u.m.a.. Comparando-se os espectros do filme e do dopante puro, é possível observar a presença de fragmentos de massas entre 26-90 u.m.a. em ambos os espectros, o que sugere uma forte contribuição de fragmentos formados pela fragmentação da cadeia carbônica do DS. Este grupo de fragmentos corresponde àqueles observados no espectro de massa do dodecano, em que as séries dos íons $\mathrm{C}_{\mathrm{m}} \mathrm{H}_{\mathrm{n}}^{+}(\mathrm{m}=2-12$ e $\mathrm{n}=1-13)$ são claramente observadas ${ }^{27}$.

Os espectros de massa dos filmes com espessuras de 0,29 e $1,90 \mu \mathrm{m}$ apresentam perfis de fragmentação semelhantes com relação ao tipo de íons formados. Entretanto, sob as mesmas condições de análise, o filme de menor espessura apresentou uma maior contribuição de íons de $\mathrm{F}^{+}$oriundos do substrato de FTO.

A calibração da escala de massa foi realizada utilizando-se o software SIMION 3D 6.0, como descrito anteriormente. Uma forte contribuição de íons $\mathrm{H}^{+}$e picos menos intensos de $\mathrm{H}_{2}^{+}$e $\mathrm{H}_{3}^{+}$podem ser observados no espectro. A presença de fragmentos de massas maiores é claramente observada. $\mathrm{O}$ espectro de massa apresenta picos referentes a fragmentos oriundos de ambas as estruturas que compõem o filme (PPy e DS). A composição do filme foi anteriormente confirmada através de outras técnicas espectroscópicas ${ }^{28-30} \mathrm{e}$ difração de raios- $\mathrm{X}^{30}$. No espectro de massa de ambos os materiais, não são observados fragmentos de massas superiores a 100 u.m.a., possivelmente devido à reneutralização e recaptura das espécies, processos altamente eficientes em superfícies. Esses processos são mais prováveis para massas mais pesadas, devido ao maior tempo que essas espécies levam para dessorver da superfície.

Para se obter uma maior compreensão dos mecanismos que levam à dessorção iônica em filmes de PPy/DS, devido à interação 


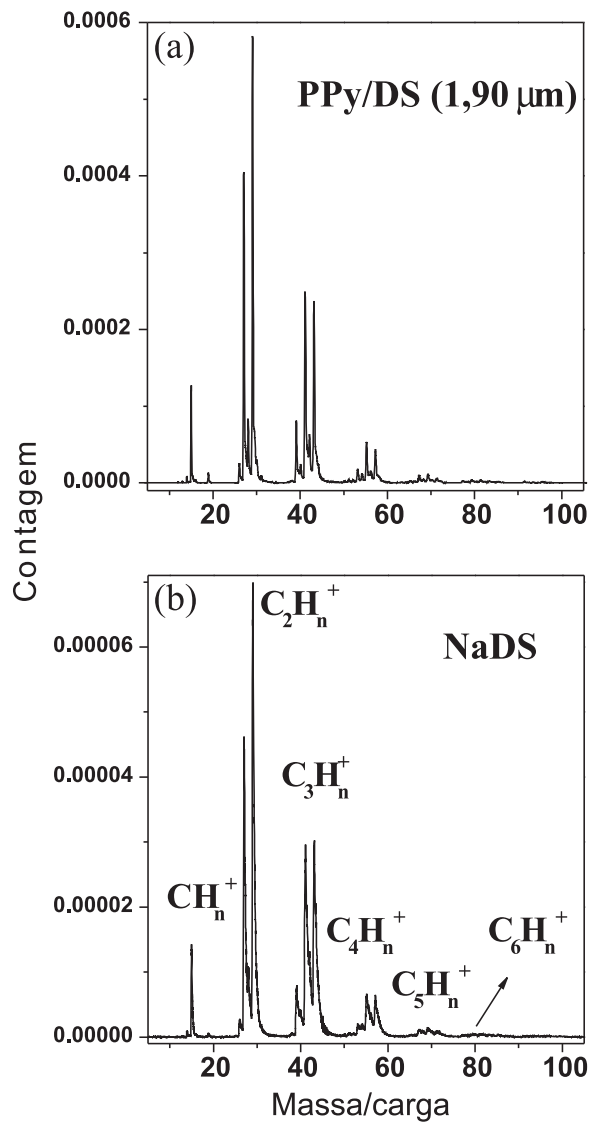

Figura 1. Espectros de massa na faixa de 5-100 u.m.a. para (a) filme de PPy/ DS (40 min) e (b) dodecilsulfato de sódio (NaDS)

com elétrons de alta energia, foram realizadas medidas com diferentes energias do feixe de elétrons incidentes (primários). As áreas dos picos foram calculadas e divididas pela área total do espectro, fornecendo o rendimento iônico relativo dos fragmentos do material. As curvas de rendimento iônico de alguns fragmentos são apresentadas na Figura 2, em função da energia do feixe de elétrons. A curva apresentada na Figura 2a é referente ao fragmento de razão massa/ carga 27, que pode ser oriundo tanto da fragmentação do polímero $\left(\mathrm{CHN}^{+}\right)$quanto do contra-íon $\left(\mathrm{C}_{2} \mathrm{H}_{3}^{+}\right)$. Entretanto, o perfil da curva de rendimento desse pico sugere uma maior contribuição do frag-

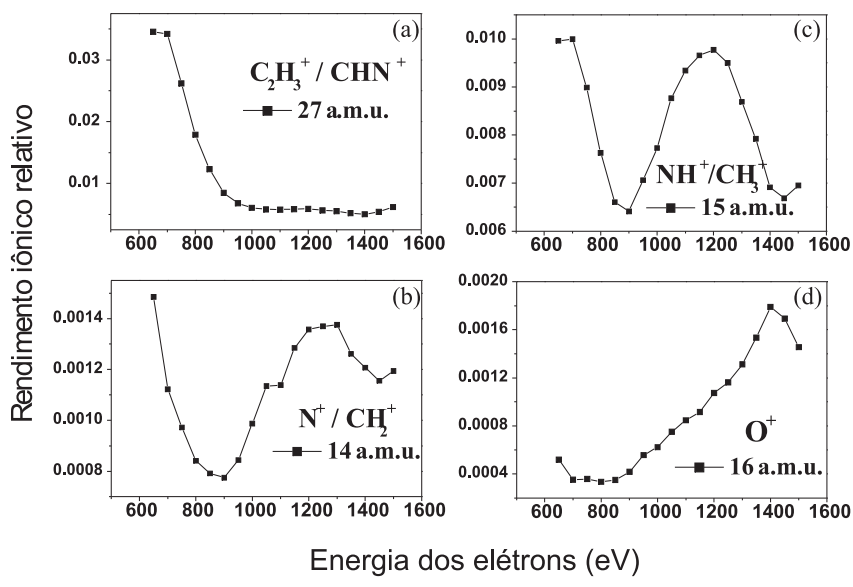

Figura 2. Curvas de rendimento iônico relativo obtidas em função da energia do feixe de elétrons incidentes para os fragmentos de massa (a) 27 u.m.a., (b) 14 u.m.a., (c) 15 u.m.a. e (d) 16 u.m.a. oriundos do filme de PPy/DS mento originário do contra-íon, devido à presença de um único máximo na curva de rendimento em torno de $650 \mathrm{eV}$. Esse tipo de curva de rendimento iônico também foi observado para os picos referentes aos fragmentos das séries de hidrocarbonetos de massas maiores. $\mathrm{O}$ perfil da curva sugere que esses fragmentos são gerados, principalmente, devido às quebras causadas por processos Auger decorrentes da excitação/ionização da camada K (elétron 1s) do átomo de carbono. Em geral, a seção de choque de ionização máxima para elétrons é alcançada quando estes apresentam energia entre 2-3 vezes o valor da energia de ionização do caroço ${ }^{31}$. Como a borda $1 \mathrm{~s}$ do átomo de carbono ocorre em aproximadamente $280 \mathrm{eV}$, a energia necessária para ocasionar o correspondente processo de ionização encontra-se entre 560 e $840 \mathrm{eV}$.

Este perfil de curva também foi observado para o pico de razão massa/carga 32, ou seja, para o fragmento $\mathrm{S}^{+}$, que pode estar associado a processos na borda $2 \mathrm{~s}$ do átomo de $\mathrm{S}(\sim 230 \mathrm{eV})$, uma vez que a faixa de energia utilizada neste trabalho não cobre as bordas 2p ( 160 eV) e 1s ( 2400 eV) do enxofre.

As curvas (b) e (c) da Figura 2 são semelhantes entre si e apresentam dois máximos, sendo o primeiro em torno de $650 \mathrm{eV}$ e o segundo em torno de $1200 \mathrm{eV}$. Neste caso, os máximos de rendimento iônico podem estar associados à fragmentação causada por processos de camada interna dos átomos de $\mathrm{C}$ e $\mathrm{N}$, respectivamente, uma vez que a borda $1 \mathrm{~s}$ do átomo de nitrogênio ocorre em torno de $400 \mathrm{eV}$. A presença de máximos de rendimento iônico em energias diferentes sugere que para os fragmentos com relação massa/ carga 14 e 15, além da contribuição dos fragmentos das séries de hidrocarbonetos oriundas do DS, tem-se também a contribuição de fragmentos contendo nitrogênio oriundo do polímero.

$\mathrm{Na}$ curva (d) da Figura 2, é possível observar um máximo em $1400 \mathrm{eV}$ que pode estar associado ao processo de camada interna do átomo de oxigênio, já que a borda 1s do oxigênio ocorre em torno de $530 \mathrm{eV}$.

A faixa de energia de 650-1500 eV apresentou curvas com máximos que demonstram favorecimento da formação dos fragmentos que contêm principalmente $\mathrm{C}, \mathrm{N}$ e $\mathrm{O}$, uma vez que nesta faixa de energia pode-se acessar a borda 1s destes átomos. Entretanto, as demais faixas de energia estudadas apresentaram perfis com comportamento constante ou crescente em função da energia dos elétrons primários.

Os fragmentos presentes nos espectros de massa do PPy/DS foram os mesmos independentemente da energia dos elétrons incidentes utilizados para ocasionar a fragmentação. Na faixa de energia empregada, é possível induzir processos de valência e de camada interna, entretanto, observa-se que é possível favorecer o rendimento iônico de um dado fragmento, utilizando-se elétrons primários com energias de 2-3 vezes à do processo de ionização de um dos átomos que participam da ligação a ser quebrada. Assim, nas energias relativas aos processos de camada interna, acredita-se que os fragmentos são formados, principalmente, pelo mecanismo ASID. Estudos de ESID de filmes de PMMA na faixa de 350 a 1200 eV mostraram um máximo de rendimento iônico em torno de 500-600 eV, que foi interpretado em termos do mecanismo ASID referente à borda $1 \mathrm{~s}$ do átomo de carbono ${ }^{32}$.

Estudos de degradação de filmes de PPy dopados com diferentes tipos de contra-ânions têm sido realizados, utilizando-se diversas técnicas de caracterização ${ }^{11,33-36}$. Muitos destes estudos são realizados através do tratamento térmico do material ou por envelhecimento, seguido da avaliação de propriedades físicas e químicas do mesmo, tais como condutividade elétrica e eletroatividade. Chehimi e colaboradores ${ }^{33}$ realizaram estudos de degradação de PPy dopado com ânions cloreto e com p-tolueno sulfonato (TS) na forma de pó, utilizando como técnicas de análise a cromatografia 
gasosa inversa (CGI), a espectroscopia de fotoelétrons (XPS) e medidas de condutividade. A análise foi realizada antes e após envelhecimento do material e também por tratamento térmico com temperatura de $150{ }^{\circ} \mathrm{C}$. Os resultados de XPS na borda $1 \mathrm{~s}$ do $\mathrm{N}$ para o $\mathrm{PPy} / \mathrm{Cl}$ mostraram que após tratamento térmico, o polímero sofre desprotonação parcial da cadeia polimérica com aumento de ligações $\mathrm{C}=\mathrm{N}$ e também diminuição da extensão de dopagem do polipirrol. A energia superficial sofreu um decréscimo significativo após o tratamento térmico e a condutividade não sofreu grandes variações. Já o PPy/TS apresentou somente $32 \%$ de sua condutividade inicial e exibiu uma energia superficial ligeiramente maior, devido à ativação superficial por tratamento térmico.

Estudos da decomposição térmica de filmes de polipirrol são mais comuns. Uma série de filmes de polipirrol dopados com diferentes ânions complexos baseados no ligante dmit (1,3-ditiola-2tiona-4,5-ditiolato) foram anteriormente estudados pelo grupo, por curvas TG-DTG. Para o filme PPy/[Ni(dmit $\left.)_{2}\right]^{2-}$, observou-se que a etapa inicial de decomposição térmica é associada com os complexos metálicos. Foram observadas perdas de massa entre 170-360 ${ }^{\circ} \mathrm{C}$, sendo as etapas posteriores de perda entre 360-510, 520-670 e 670-800 ${ }^{\circ} \mathrm{C}$ relacionadas com a degradação do polipirrol com clivagem de produtos de alta massa molar como oligômeros ou dímeros $^{37}$. Pereira e colaboradores ${ }^{29}$ estudaram o polipirrol dopado com $\left[\mathrm{NBu}_{4}\right]\left[\mathrm{Bi}(\mathrm{dmit})_{2}\right]$ e observaram que a decomposição térmica se inicia em aproximadamente $180^{\circ} \mathrm{C}$, também relacionada ao contra-ânion. Essa temperatura foi maior do que a observada para a etapa inicial de decomposição térmica de filmes de PPy/DS, que mostraram perdas iniciais a $133{ }^{\circ} \mathrm{C}$ relacionadas ao DS. Da Cruz e colaboradores $^{38}$ encontraram temperatura inicial de decomposição térmica acima de $200{ }^{\circ} \mathrm{C}$ para o PPy dopado com [ $\left.\mathrm{NEt}_{4}\right]_{2}\left[\mathrm{Sn}(\mathrm{dmit})_{3}\right]$. Com esses resultados, sugeriu-se que a estabilidade térmica de filmes PPy/M-dmit está relacionada à estabilidade térmica dos contra-ânions do PPy, confirmando o que já fora observado para filmes dopados com outros contra-ânions.

No presente trabalho, estudos de degradação do filme de PPy/ DS foram realizados com o emprego de feixe contínuo de elétrons de $2,2 \mathrm{keV}$ e corrente de emissão de $\sim 4,5 \mu \mathrm{A}$. As curvas de degradação de alguns fragmentos são apresentadas na Figura 3, onde se encontra o rendimento iônico em função do tempo de exposição da amostra ao feixe contínuo de elétrons. O perfil das curvas apresentadas pelos fragmentos de massa 15 e 27 (Figuras 3a e 3b) mostra um decréscimo rápido do rendimento iônico com o tempo de exposição ao feixe de elétrons. Este perfil também foi observado para os demais picos que apresentaram uma maior contribuição dos fragmentos das séries de hidrocarbonetos.

O perfil da curva apresentada na Figura $3 \mathrm{c}$ foi observado para os picos atribuídos aos fragmentos de massa 9,5; 18 e 19 que correspondem, respectivamente, aos fragmentos $\mathrm{F}^{2+}, \mathrm{H}_{2} \mathrm{O}^{+}$e $\mathrm{F}^{+}$. O fragmento de massa 18 pode ser originário do "bulk", uma vez que o filme foi sintetizado em água e esta é incorporada juntamente com o ânion dentro da matriz polimérica. Os fragmentos de massa 9,5 e 19 decorrem do substrato de FTO, uma vez que este contém flúor em sua composição. O perfil de degradação destas curvas mostra um aumento do rendimento destes íons com relação ao tempo de exposição ao feixe de elétrons, o que é esperado uma vez que tanto a exposição do interior do filme quanto do substrato ao feixe de elétrons aumenta com a degradação.

$\mathrm{O}$ perfil da curva apresentada na Figura $3 \mathrm{~d}$ foi observado para os fragmentos de massa $8,12,16$ e $32\left(\mathrm{O}^{2+}, \mathrm{C}^{+}, \mathrm{O}^{+}\right.$e $\mathrm{S}^{+}$, respectivamente). Este perfil sugere um rendimento baixo para tempos curtos de exposição à radiação, sofrendo um aumento seguido de decaimento lento quando o tempo de exposição é aumentado. Este perfil sugere que o tempo de exposição contribui para a quebra de

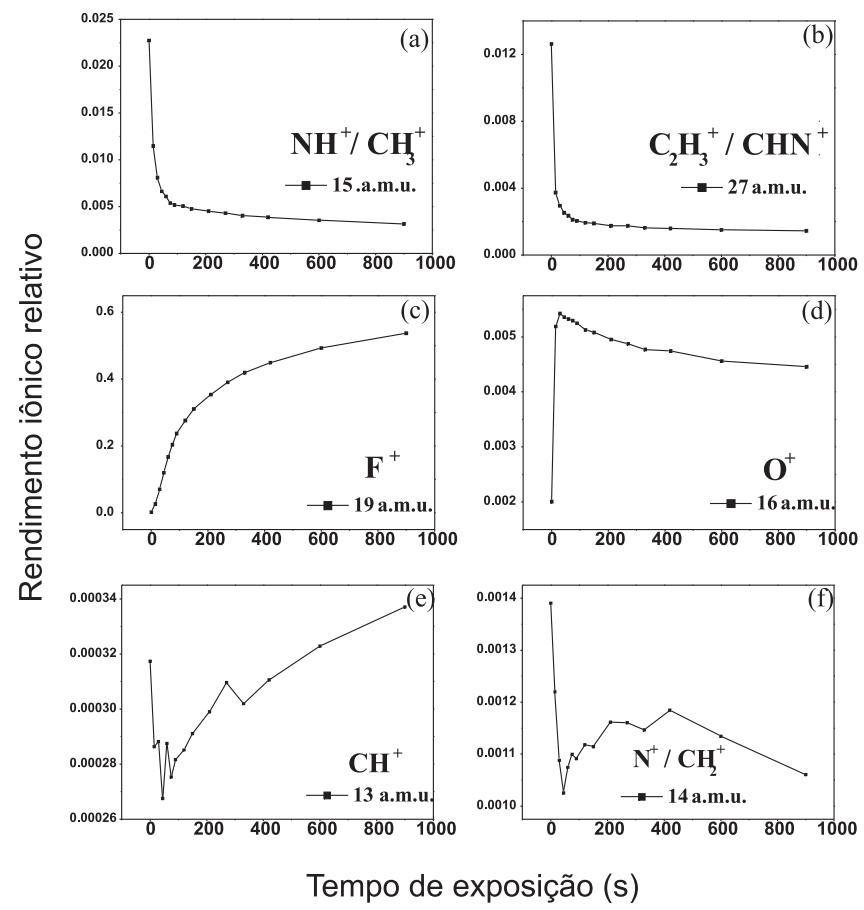

Figura 3. Curvas de degradação obtidas para os fragmentos de massas (a) 15 u.m.a., (b) 27 u.m.a., (c) 19 u.m.a., (d) 16 u.m.a., (e) 13 u.m.a. e (f) 14 u.m.a. oriundos do filme de PPy/DS, induzida por elétrons de $2,2 \mathrm{keV}$

espécies maiores, gerando estes fragmentos menores. O comportamento observado para as séries de hidrocarbonetos (Figuras 3a e 3b) corrobora esta interpretação.

A curva apresentada na Figura 3e caracteriza os fragmentos de massa 13, 31 e (50-52) e sugere uma degradação inicial das espécies presentes no filme, seguida de formação de outras espécies de mesma massa, sejam elas pré-existentes ou não. A curva dos fragmentos de massa (50-52), que foram calculados juntos, mostrou um aumento menos pronunciado que as demais, possivelmente por se tratar de massas maiores, que conseqüentemente são mais difíceis de serem observadas. O aumento de rendimento iônico da curva do fragmento $\mathrm{CH}^{+}$pode estar associado à degradação de espécies de massas maiores, originando este fragmento. A curva referente ao fragmento de massa 31 pode ser interpretada de forma similar, quando é considerado o fragmento $\mathrm{CH}_{3} \mathrm{O}^{+}$. Entretanto, existe a possibilidade de formação de espécies $\mathrm{CF}^{+}$, originárias da recombinação de espécies dessorvidas durante a degradação do filme e do substrato. A interpretação da curva de degradação dos fragmentos de massas 50-52 também é feita de forma similar, porém neste caso o perfil da curva não foi tão acentuado, podendo estar relacionado à formação de novas espécies.

Na Figura 3f, é apresentado um último perfil de curva de degradação, observado para o fragmento de massa 14, que se apresentou diferente dos demais. Como para essa massa temos a possibilidade dos fragmentos $\mathrm{N}^{+} \mathrm{e} \mathrm{CH}_{2}^{+}$, provavelmente ocorre uma diminuição de rendimento no início da degradação, principalmente devido à eliminação dos fragmentos de $\mathrm{CH}_{2}^{+}$que, como os demais hidrocarbonetos, apresentam uma diminuição brusca de rendimento num curto período de exposição ao feixe de elétrons. Após a eliminação desta espécie, ocorre um aumento, seguido de diminuição, que pode estar relacionado com a formação e eliminação do fragmento $\mathrm{N}^{+}$.

\section{CONCLUSÕES}

Estudos de dessorção iônica estimulada por elétrons de filmes 
de PPy/DS foram realizados, utilizando-se uma faixa de energia de 650-2900 eV e um espectrômetro de massa do tipo tempo-de-vôo. Os espectros de massa obtidos mostraram uma forte contribuição de fragmentos do contra-ânion, confirmando sua incorporação na matriz polimérica, conforme verificado em trabalhos anteriores por FTIR e XPS. As curvas de rendimento iônico apresentaram máximos em torno de 600, 1200 e $1400 \mathrm{eV}$, indicando que o mecanismo de dessorção iônica estimulada por processo Auger (ASID) exerce papel importante na formação dos fragmentos analisados, na faixa de energia entre 650-1500 eV. As curvas de degradação indicaram rápida diminuição do rendimento dos fragmentos de hidrocarbonetos pesados, aumento do rendimento dos fragmentos originários do substrato e do bulk do material, aumento do rendimento de espécies menores e perfis de degradação sugerindo formação de novas espécies.

\section{MATERIAL SUPLEMENTAR}

O diagrama do sistema experimental utilizado para a realização dos experimentos de ESID ("Electron Stimulated Ion Desorption") e de degradação dos filmes de polímeros condutores é apresentado na Figura 1S e as atribuições do picos de massas entre 1 e 100 u.m.a. são apresentadas na Figura 2S.

\section{AGRADECIMENTOS}

C. Arantes agradece à CAPES pela bolsa de mestrado e os autores agradecem o apoio financeiro da FAPERJ, CNPq e Instituto do Milênio de Materiais Complexos (IMMC).

\section{REFERÊNCIAS}

1. De Paoli, M. A.; Zanelli, A.; Mastragostino, M.; Rocco, A. M.; J. Electroanal. Chem. 1997, 435, 217.

2. Song, H. K.; Palmore, G. T. R.; Adv. Mater. 2006, 18, 1764.

3. Kim, J. H.; Sharma, A. K.; Lee, Y. S.; Mater. Lett. 2006, 60, 1697.

4. Gupta, N.; Sharma, S.; Mir, I. A.; Kumar, D.; Journal of Scientific \& Industrial Research 2006, 65, 549.

5. Peres, R. C. D.; Pernaut, J. M.; De Paoli, M. A.; J. Polym. Sci., Part A: Polym. Chem. 1991, 29, 225.

6. Dai, T. Y.; Yang, X. M.; Lu, Y.; Nanotechnology 2006, 17, 3028.

7. Omastova, M.; Pionteck, J.; Trchova, M.; Synth. Met. 2003, 135, 437.

8. Stejskal, J.; Omastova, M.; Fedorova, S.; Prokes, J.; Trchova, M.; Polymer 2003, 44, 1353.
9. Minto, C. D. G.; Vaughan, A. S.; J. Mater. Sci. 1995, 30, 6028.

10. Wolszczak, M.; Kroh, J.; Abdelhamid, M. M.; Radiat. Phys. Chem. 1995, 45,71 .

11. Gazotti ,W. A.; Juliano, V. F.; De Paoli, M. A.; Polym. Degrad. Stab. 1993, $42,317$.

12. Rapta, P.; Neudeck, A.; Bartl, A.; Dunsch, L.; Electrochim. Acta 1999, 44, 3483.

13. Dunsch, L.; Rapta, P.; Neudeck, A.; Bartl, A.; Peters, R. M.; Reinecke, D.; Apfelstedt, I.; Synth. Met. 1997, 85, 1401.

14. Adachi, A.; Yamauchi J.; Synth. Met. 1995, 73, 101.

15. Tinone, M. C. K.; Tanaka, K.; Maruyama, J.; Ueno, N.; Imamura, M.; Matsubayashi, N.; J. Chem. Phys. 1994, 100, 5988

16. Simons, J. K.; Frigo, S. P.; Taylor J. W.; Rosenberg R. A.; J. Vac. Sci. Technol., A 1994, 12, 681.

17. Rocco, M. L. M.; Pontes, F. C.; Faraudo, G. S.; de Souza, G. G. B.; Quim. Nova 2004, 27, 160.

18. Tanaka, K.; Tinone, M. C. K.; Ikeura, H.; Sekiguchi, T.; Sekitani, T.; Rev. Sci. Instrum. 1995, 66, 1474.

19. Ahn, H.; Oblas, D. W.; Whitten, J. E.; Macromolecules 2004, 37, 3381.

20. Sevil, U. A.; Guven, O.; Kovacs, A.; Slezsak I.; Radiat. Phys. Chem. 2003, $67,575$.

21. Xu, H. S.; Cheng, Z. Y.; Zhang, Q. M.; Wang, P. C.; MacDiarmid, A. G.; Synth. Met. 2000, 108, 133.

22. Rocco, M. L. M.; Weibel, D. E.; Roman, L. S.; Micaroni, L.; Surf. Sci. 2004, 560, 45.

23. Wernet, W.; Wegner, G.; Makromolekulare Chemie - Macromolecular Chemistry and Physics 1987, 188, 1465.

24. Mendes, L. A. V.; Dissertação de Mestrado, Universidade Federal do Rio de Janeiro, Brasil, 2006.

25. Wiza, J. L.; Nucl. Instrum. Methods Phys. Res., Sect. B 1979, 162, 587.

26. Dahl, D. A., ed;. SIMION 3D Version 6.0, User's Manual, Idaho National Engineering Laboratory, 1995.

27. http://webbook.nist.gov/cgi/cbook.cgi?ID=C112403\&Units=SI\&Mask= 200\#Mass-Spec, acessada em Novembro 2006.

28. Arantes, C.; Dissertação de Mestrado, Universidade federal do Rio de Janeiro, Brasil, 2006.

29. Pereira, R. P.; Wardell, J. L.; Rocco, A. M.; Synth. Met. 2005, 150, 21.

30. Nalwa, H. S., ed.; Handbook of organic conductive molecules and polymers, John Wiley \& Sons: New York, 1997, vol. 3.

31. Chang, C. C.; Surf. Sci. 1971, 25, 53.

32. Rocco, M. L. M.; Weibel, D. E.; Pontes, F. C.; Pinho, R. R.; Faraudo, G. S.; de Souza, G. G. B.; Polym. Degrad. Stab. 2003, 80, 263

33. Chehimi, M. M.; Abdeljalil, E.; Synth. Met. 2004, 145, 15.

34. Otero, T. F.; Boyano, I.; Electrochim. Acta 2006, 51, 6238.

35. Ricks-Laskoski, H. L.; Buckley, L. J.; Synth. Met. 2006, 156, 417.

36. Papathanassiou, A. N.; Grammatikakis, J.; Sakellis, I., Sakkoupolos, S.; Vitoratos, E.; Dalas, E.; Appl. Phys. Lett. 2005, 87, 154107.

37. da Cruz, A. G. B.; Wardell, J. L.; Rocco, A. M.; Synth. Met. 2007, 157, 80.

38. da Cruz, A. G. B.; Wardell, J. L.; Rocco, A. M, Synth. Met. 2006, 156, 396; da Cruz, A. G. B; Tese de Doutorado, Universidade Federal do Rio de Janeiro, Brasil, 2006 
DESSORÇÃO IÔNICA E DEGRADAÇÃO DE FILMES DE POLIPIRROL DOPADO COM DODECILSULFATO INDUZIDAS POR ELÉTRONS DE ALTA ENERGIA

Caroline Arantes e Maria Luiza M. Rocco*

Departamento de Físico-Química, Instituto de Química, Universidade Federal do Rio de Janeiro, Cidade Universitária, Ilha do Fundão, 21941-590 Rio de Janeiro - RJ, Brasil

Antonio Gerson Bernardo da Cruz e Ana Maria Rocco

Escola de Química, Universidade Federal do Rio de Janeiro, Cidade Universitária, Ilha do Fundão, 21949-900 Rio de Janeiro - RJ, Brasil

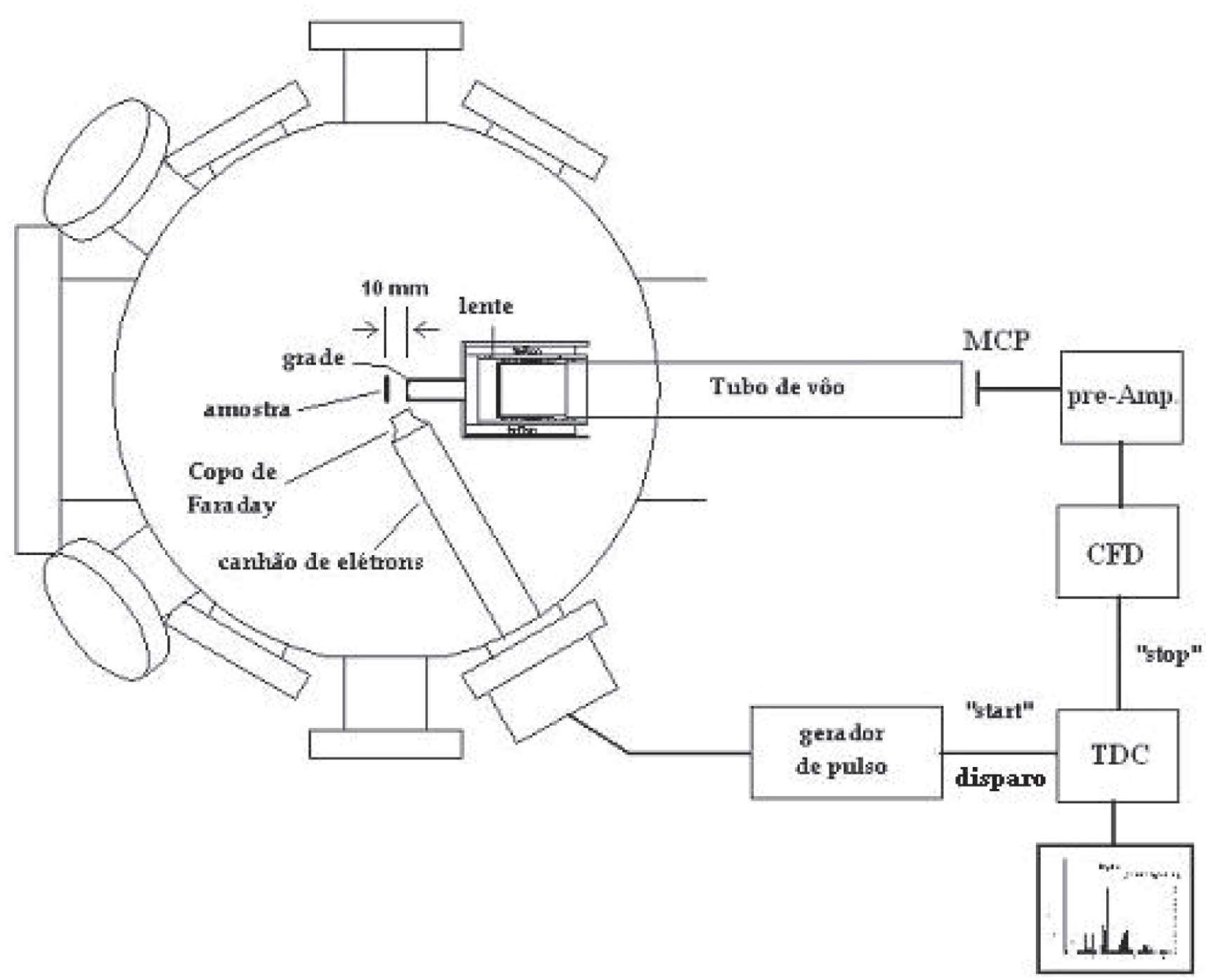

Figura IS. Diagrama do arranjo experimental utilizado no estudo da interação de elétrons com polímeros condutores através da técnica de ESID 

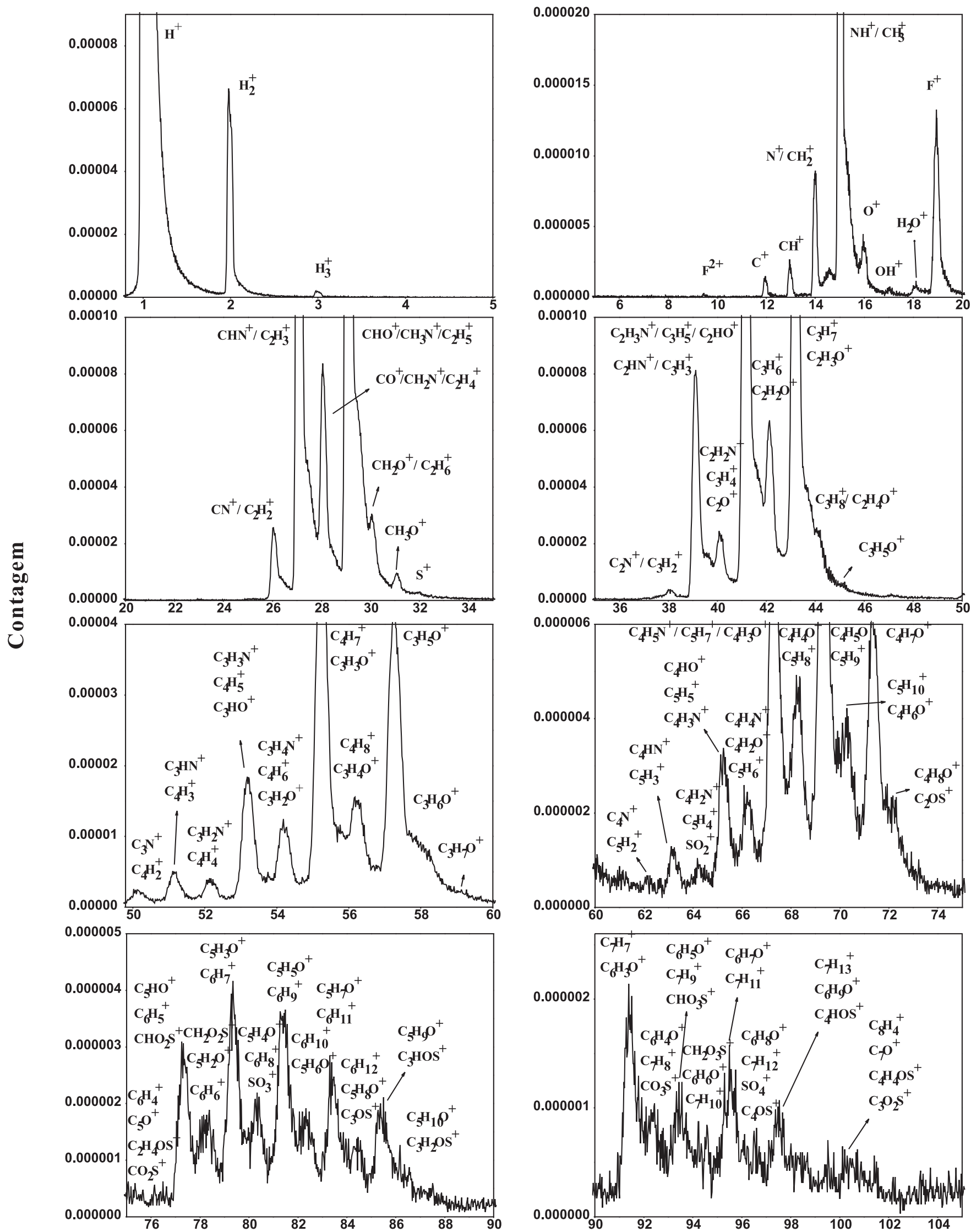

Massa/carga (u.m.a.) 